\title{
Editorial
}

\section{The Development of VGG in Japan}

\author{
Masao Takayasu, M.D.*
}

Nowadays, there can not be any clinic without electrocardiograph and moreover VGG has also become popular for daily physical examination. But in our country, the progress and popularization have not progressed rapidly, in spite of being one of the countrics in which VGG was first discovered. In the ensuing years, the studies of VGG have become more popular. There are problems about the lead method, namely the placement of electrodes; and many different methods have been used. For examples, methods by Duchosal, Grishman, Kimura and others were used in a combination of bipolar leads, and methods by Milovanovich and Wilson-Burch in a combination of unipolar, or unipolar and bipolar leads. In 1939, N. Kimura, independent of Schellong, reported about the VCG with his lead method for the first time. For several years, little progress was made. Then from about 1950 many investigators, T. Sano, Toyoshima and Toyama and others, studied clinically and obtained results with Grishman's method. And as a fundamental study, Toyoshima reported about the "poliograph" in 1946. It is a more ideal lead method but troublesome to use, as it requires special oscilloscope. Since then, he has studied with his reconstruction method and has made many investigations.

On the other hand, as an ideal lead system for spatial VGG the following postulates must be satisfied;

1) 3 orthogonal components of electromotive forces of the heart parallel to $X, Y$ and $Z$ axis of the body must be led with minimum distortion,

2) preferably no equalization factor for any axis is needed,

3) simplicity of the electrode system and procedure of its application is desired.

In 1953, McFee and Johnston reported about the lead field concept. Then the orthogonal lead systems, which are better fit to the above mentioned first postulate, were widely studied and several lead systems were presented by Schmidt, Frank, Helm and others. We have also studied how to introduce this concept in clinical use, and in 1955 presented

\footnotetext{
* Professor of Internal Medicine, Mie Prefectural University School of Medicine, Tsu.
} 
a new orthogonal system which consists of 2 pairs of large electrode plates: one pair is on the right and the left side of the chest, one pair on the back and the front of the chest as the heart is covered. And for $\mathrm{Y}$ lead, $a V_{F}$ was employed. In this method, the lead fields between 2 electrodes are parallel and uniform, so the electromotive forces in this field will be led without distortion even if the dipoles move within this field. In this sense, this lead system may be more ideal.

The projection of the VGG loop by this lead method to the lead axis of the chest lead, quite resembles the ECG of the chest lead. Then for the practical use we studied the VGG lead system, which employs Wilson's V chest lead, but modified the electrode position. This was named $\mathrm{V}$ lead. In this $\mathrm{V}$ lead the electrode of $\mathrm{Vx}$ was placed on the middle line of anterior- and mid-axillary line, at the level of the 5 th rib on mammary line, and the $\mathrm{Vz}$ was placed on the left sternum border at the same level. And the horizontal loop was given with $\mathrm{Vx}$ and $\mathrm{Vz}$. And for $Y$ axis, the $V_{F}$ was used.

Recently, as a lead system for VGG, Frank's method has been mostly used in Japan. But for the progress of VGG, the data obtained by several authorities must be collected and compared, so the unification of the lead method may bc obtained, and a more ideal lead method, if there is one, may be found.

In the symposium "Discussion between Electrocardiographer and Vectorcardiographer" at the annual meeting of the Japanese Circulation Society, April, 1962, discussion was held about VGG and the conclusion was the same as the above. And for the future progress of VCG, it would be desirable not only to compare the excellence of VGG with EGG, but to develope the prominent points of VGG itself. Moreover, it is also necessary to study more about $\mathrm{P}$ and $\mathrm{T}$ loop. 